\title{
EFFECTIVENESS OF CONVENTIONAL ANTIBIOTICS IN SALMONELLA TYPHI POSITIVE BLOOD CULTURES
}

\author{
Aftab Ahmad Khan, Rizwan Uppal, Gul E Rehan, Farhat Khurshid, Khurshid Ahmad, Hina Zaib \\ Islamabad Diagnostic Centre, Islamabad - Pakistan
}

\begin{abstract}
Objective: To determine the pattern of resistance of Salmonella Typhi strains to multiple antibiotics in Islamabad and adjoining areas.

Material and Methods: A retrospective study conducted from audit of 15 months (Jan' 2018 to March' 2019), recruiting those patients whose blood cultures were found positive for Salmonella Typhi at Islamabad Diagnostic Center. We analyzed 100 samples and positive cultures were then assessed for sensitivity by using various antibiotics and pattern of resistance was analyzed.
\end{abstract}

Results: Majority of positive patients were male (63\%) and children (65\%). Sensitivity to the conventional antibiotics was found to be more in children. In all the positive isolates, $89 \%$ were sensitive to the first line treatment options.Among them, 96.6\%were showing sensitivity to third generation Cephalosporins. However, a few cases were having Extensively Drug Resistant (XDR) patterns.

Conclusion: Despite the fact that XDR are emerging and indicating an alarming situation, more than $80 \%$ patients were responding to the conventional therapy, making it a dire need for up-gradation of antibiograms, so that the side effects of second line and advanced antibiotic can be avoided, especiallyin pediatric population.

Keywords: Non-resistant typhoid fever, sensitivity patterns

This article may be cited as: Khan AA, Uppal R, Rehan GE, Khurshid F, Ahmad K, Zaib H. Effectiveness of conventional antibiotics in Salmonella Typhi positive blood cultures. J Med Sci 2020 October;28(4):318-322

\section{INTRODUCTION}

Bacteria Salmonella enterica serovar Typhi (S. Typhi) causes Typhoidal fever and transmission occurs via the orofecal route. ${ }^{1,2,3}$ Approximately 17 million cases of enteric fever occurred globally in 2015,mostly in Asia and sub-Saharan Africa where the largest burden and incidence was found in South Asia. In 2015, 178,000 deaths were reported worldwide due to Typhoid fever. ${ }^{4,5}$ Asia has the highest frequency rate of 274 cases per 100,000 population and is five times greater than the second highest, Latin America. Pakistan has the highest incidence (451.7 per 100,000 persons/year) of typhoid fever followed by India (214.2 per 100,000 persons/year). ${ }^{6}$ Before the introduction of antimicrobials, death occurred in about $33 \%$ of patients having typhoid fever in developing countries and up-to $10 \%$ of cases in developed countries. ${ }^{7}$ With the advent of antimicrobialsin 1950s, the fatalities were reduced to less than $2 \%$ but the emergence of resistant strains in

\section{Correspondence}

Dr. Gul E Rehan

Associate Hematologist, Islamabad Diagnostic Center, Islamabad - Pakistan.

Email: rehangule@gmail.com

Cell: +92-332-9320744

Date received: $21-04-2020$

Date revised: $\quad 07-08-2020$

Date accepted: $10-08-2020$ high-burden countries has been a daunting concern in recent years. ${ }^{8}$

Several mechanisms are involved in S. Typhi antibiotic resistance including inactivation of drug, alteration of the target site and active efflux. These occur due to multiple reasons which include injudicious use of antibiotics, use of antibiotics in animal feed to promote the growth of animals and in veterinary medicine to treat bacterial infections in those animals ${ }^{9}$.

Emergence of decreased susceptibility to conventional therapies has also begun in high-burden regions such as South and Southeast Asia. ${ }^{10}$ Cases of XDR Typhoidal Salmonella are also emerging in these areas, which is defined as strains resistant to Ampicillin, Chloramphenicol, Trimethoprim Sulfamethoxazole, third generation Cephalosporins and Fluoroquinolones. Due to increasing resistance to standard therapies, new antimicrobials such as Carbapenems, Tigecycline, and Azithromycin are being monitored as potential treatment options. ${ }^{11}$ Asia is one of the continents with a high isolation frequency of S.Typhi displaying XDR phenotype. In a surveillance study conducted in Hyderabad, Pakistan over a 10-month period between 2016 and 2017, health authorities detected more than 800 cases of extensively drug-resistant typhoid in this city alone. ${ }^{12}$ 
Interesting fact found in a few studies is the re-emergence of the sensitivity of S.Typhi to the initial conventional therapy i.e. Chloramphenicol, Ciprofloxacin, Amikacin and Ampicillin was $96 \%, 88 \%, 84 \%$ and $48 \%$ respectively. ${ }^{1}$

\section{MATERIALS AND METHODS}

The data of blood cultures of patients referred to Islamabad Diagnostic Center from Jan' 2018 to March' 2019 was retrieved from medical record. Data included demographics, clinical presentation, investigations and diagnosis.

Blood culture samples were collected under aseptic measures and poured aseptically into blood culture bottles and incubated in Versa Trek Blood Culture System according to manufacturer's instructions. From the positive blood culture bottles, a few drops are withdrawn using strict aseptic precautions and sub cultured on Blood Agar and MacConkey Agar. Plates were incubated for overnight at $37 \stackrel{\circ}{ }$. Oxidase test was done on non lactose fermenting (NLF) colonies and motility was checked microscopically. Motile, gram negative rods that were oxidase negative were then subjected to biochemical testing with Indole, Methyl red, VP and citrate (-,+,-,+ respectively). Final identification was done on Analytical Profile Index (API) strips.

Discrete S.Typhi isolates were tested for susceptibility to various antimicrobial agents by quality controlled disk diffusion techniqueon Mueller-Hinton Agar. The antibiotics (Oxoid Ltd., Basingstoke, United Kingdom) screened included; Ampicillin $(10 \mu \mathrm{g})$, Amoxicillin-Clavulanic acid $(30 \mu \mathrm{g})$, Cefotaxime $(30 \mu \mathrm{g})$,Cefixime $(5 \mu \mathrm{g})$, Ciprofloxacin $(5 \mu \mathrm{g})$, Levofloxacin , Moxifloxacin, Chloramphenicol $(30 \mu \mathrm{g})$, Cotrimoxazole $(25 \mu \mathrm{g})$ and Azithromycin $(15 \mu \mathrm{g})$. The results were interpreted as sensitive, intermediate or resistant in accordance withClinical \& Laboratory Standards Institute (CLSI guidelines)2018.Data was collected with standardized forms and transferred daily to a server. The descriptive data was analyzed using SPSS version 25.0.

\section{RESULTS}

A total of 100 samples positive for S.Typhi were included in this study. About $63 \%$ patients were male and $37 \%$ were female (figure 1). Sixty five percent of patients of the study were below the age of 19 years and 35\% were adults (figure 2).

Sensitivity patterns were analyzed as per WHO recommendations. According to $\mathrm{WHO}$, (disease outbreak news) Non Resistant Typhoid fever is defined as the onewhich issensitive to the first line of treatment (Chloramphenicol, Ampicillin, Trimethoprim-Sulfamethoxazole) and third generation Cephalosporins, with or without resistance to second line drugs (Fluoroquinolones). Among all the isolates which were found positive for S. Typhi, 89\% were sensitive to anyone or multiple of the first line treatment options (figure 3).

The sensitivity of the isolates was found to be more in children (64\%) as compared to adults (36\%). (figure 4$)$. They were then assessed separately for each one of the first line of treatment options and it was discovered that most of them i.e. 86 (96.6\%) out of the total 89 sensitive patients were showing sensitivity to third generation Cephalosporin. Sensitivitypercentages for Chloramphenicol, Ampicillin, Trimethoprim-Sulfamethoxazole were 51.7\%, $64 \%$ and $56.2 \%$ respectively. (figure 5 )

Sensitivity was also determined for their response to second line drugs (Fluoroquinolones) and was found out that $13(14.6 \%)$ of total 89 patients, who were declared as having Non Resistant Typhoid fever were resistant to second line drugs (Fluoroquinolones)(figure 6).

As per definition by WHO, XDR is defined as the strains which are resistant to all of the recommended treatment options for S. Typhi (Chloramphenicol, Ampicillin, Trimethoprim-Sulfamethoxazole, third generation Cephalosporins, second line drugs) and our data analysis

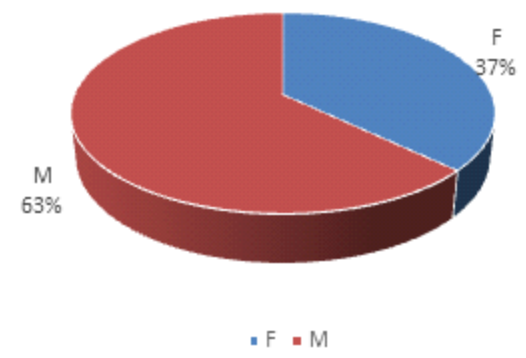

Fig 1: Gender wise distribution of disease

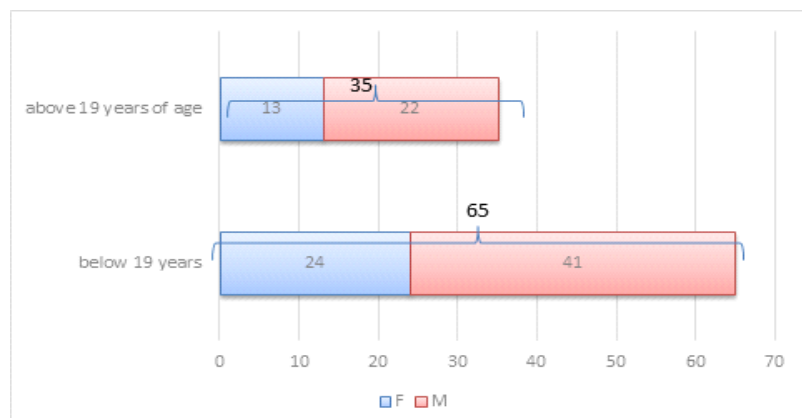

Fig 2: Age and gender wise distribution of disease

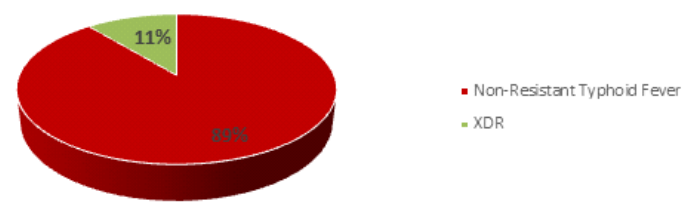

Fig 3: Pattern of response to conventional therapy 


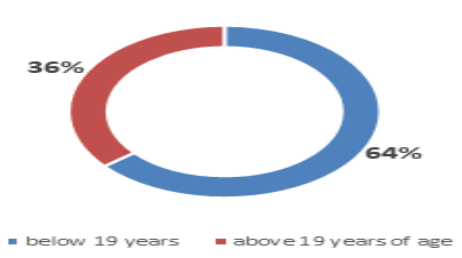

Fig 4: Age wise assessment of sensitivity to therapy

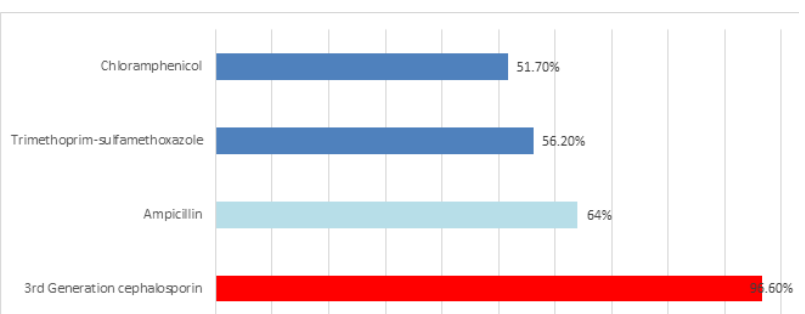

Fig 5: Assessment of sensitivity percentages of non resistant typhoid fever

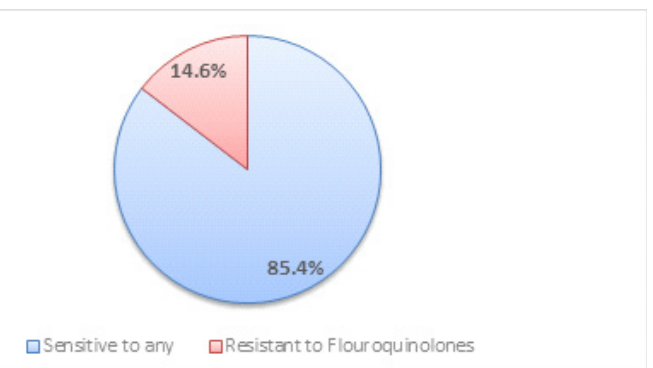

Fig 6: Percentage of non resistant typhoid fever, resistant to Fluoroquinolones

Cases with XDR pattern

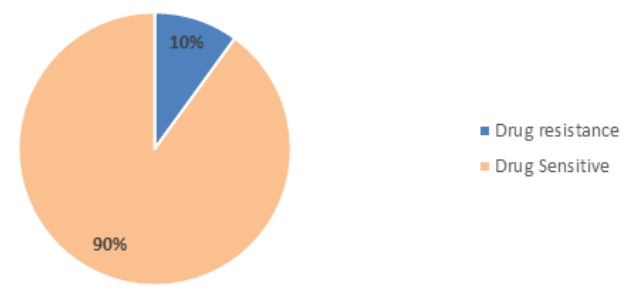

Fig 7: Percentage of cases with XDR

showed that $10 \%$ of cases were having XDR.(figure 7 )

\section{DISCUSSION}

Typhoid fever continues to be a public health problem in Pakistan being exacerbated by emerging resistance to antibiotics that were effective earlier. Wide variation in the sensitivity pattern of various strains is circulating in different geographic regions in Pakistan making it a dire need to assess the sensitivity of typhoid bacilli to antibiotics before instituting therapy.
In our study majority of the patients positive for Salmonella Typhi were male and children and results are consistent with a study conducted at Agha Khan University Hospital, Pakistan ${ }^{14}$ as well as those conducted in India and Bangladesh. ${ }^{15}$

According to the WHO criteria, $89 \%$ of our positive blood culture samples were included in the category of non-resistant typhoid fever. These samples were sensitive to any one or more of the recommended therapies in this criterion. ${ }^{11}$

After assessing separately each of these drugs classes, it was revealed that $96.6 \%$ patients (86 out of the total 89 non resistant typhoid fever patients) were sensitive to third generation Cephalosporin. This result shows that the patients, majority of whom are children can be treated effectively by this class of drugs (third generation of Cephalosporin).This percentage is quite different from a study conducted recently in southern Pakistan which shows a sensitivity pattern of almost $60 \%{ }^{16}$

Although these non-resistant typhoid cases are sensitive to first line drugs and third generation Cephalosporins, our study found out that $13 \%$ of these were surprisingly resistant to second line drugs (Fluoroquinolones) which proves the erroneous use of this class of drug. ${ }^{17}$ In corresponding to this finding, a systematic review conducted by Carl D. Britto et al shows that in Asia resistance to Fluoroquinolones continued to increase during 2001 to 2005 period by about $20 \% .{ }^{18}$ Fluoroquinolones use in children is recommended in limited conditions by FDA. However these are still widely used by general practitioners for Salmonella Typhi ultimately leading to long term side effects as well as emergence of further resistant strains.

The cost effectiveness and over all safety of third generation Cephalosporins make them the preferred medicine, especially in children.This way the devastating side effects of quinolones like arthropathy and cartilaginous damage can be avoided. ${ }^{19,20}$ Moreover if initially a patient is given Fluoroquinolones, there will be a delay in treatment response as well as increase incidence of complications due to the presence of more than $10 \%$ resistant cases.

The other recommended drugs for non resistant typhoid fever are Ampicillin, Trimethoprim-Sulfamethoxazole and Chloramphenicol and the sensitivity percentages in our patients were $64 \%, 56.2 \%$ and $43 \%$ respectively. Chloramphenicol causes bone marrow aplasia in children 
Effectiveness Of Conventional Antibiotics In Salmonella Typhi Positive Blood Cultures.

so its use is not encouraged in young population. Though

Penicillin class and Trimethoprim-Sulfamethoxazole can be safely used but our study revealed significant percentage of resistance to them and there is an increased chance of treatment failure. Contrary to our study, another study conducted in India by JK Bhatia et al showed sensitivity of $96 \%$ to Chloramphenicol and $48 \%$ to Ampicillin. ${ }^{21}$

Another alarming situation, due to the misuse of drugs is the emergence of extended drug resistant strains and results show that those patients comprise almost $10 \%$ of the considered population. These patients only show sensitivity to Azithromycin.

Being a massively typhoid affected population of South Asia, immediate and sagacious steps are needed on national grounds to decrease the disease incidence by its prompt diagnosis and appropriate treatment strategies.

Interesting finding from our study is the recent re-emergence of the pattern of sensitivity of strains to the initially used drugs. These findings go in parallel with a study conducted for assessing the re emergence of susceptibility to conventionally used drugs for S.Typhi. ${ }^{22}$

Over the years, as the patients were switched to more extensive and advanced classes of drugs, the relatively cheaper and safer drugs are now again showing adequate and impressive sensitivity patterns. Compilation of data from different geographic zones of the country is required to further substantiate our findings for better disease management and avoidance of complications.

\section{CONCLUSION}

The conventional anti-typhoidal treatment, i.e. third-generation Cephalosporins can be used as an effective empirical therapy for treating typhoid fever cases in our setting. However the emergence of XDR pattern needs attention at national level so that the disease related morbidity and mortality can be reduced.Analysis of larger patient population from different epidemiological areas will be highly effective in formulation of policies for the management of typhoid fever cases in our country.

\section{LIMITATIONS}

Main limitation of the study is that it is conducted at a single center.

\section{REFERENCES}

1. Gibani M, Britto C, Pollard A. Typhoid and paratyphoid fever [Internet]. National Center for Biotechnology Infor- mation. 2020 [cited 24 April 2020]. Available from: https:// www.ncbi.nlm.nih.gov/pmc/articles/PMC6319573/

2. Wangdi T, Winter S, Bäumler A. Typhoid fever. Gut Microbes. 2012;3(2):88-92.

3. Habte L, Tadesse E, Ferede G, Amsalu A. Typhoid fever: clinical presentation and associated factors in febrile patients visiting Shashemene Referral Hospital, southern Ethiopia. BMC Research Notes. 2018;11(1).

4. Salmonella: A review on pathogenesis, epidemiology and antibiotic resistance [Internet]. Taylor \& Francis. 2020 [cited 24 April 2020]. Available from: https://www. tandfonline.com/doi/full/10.1080/21553769.2015.10512 43

5. Antillón M, Warren J, Crawford F, Weinberger D, Kürüm E, Pak $G$ et al. The burden of typhoid fever in low- and middle-income countries: A meta-regression approach. PLOS Neglected Tropical Diseases. 2017;11(2):e0005376.

6. Siddiqui F, Rabbani F, Hasan R, Nizami S, Bhutta Z. Typhoid fever in children: some epidemiological considerations from Karachi, Pakistan. Int J Infect Dis. 2006;10(3):215-222.

7. Jong E, Stevens D, Netter F. Netter's infectious diseases. Philadelphia, PA: Elsevier/Saunders; 2012.

8. Bloom D, Black S, Salisbury D, Rappuoli R. Antimicrobial resistance and the role of vaccines. Proceedings of the National Academy of Sciences. 2018;115(51):1286812871.

9. Ugboko H, De N. Mechanisms of Antibiotic resistance in Salmonella typhi. Int J Curr Microbiol App Sci. 2014; 3(12): 461-476.

10. Klemm EJ, Shakoor S, Page AJ, Qamar FN, Judge K, et al. Emergence of an Extensively Drug-Resistant Salmonella enterica Serovar Typhi Clone Harboring a Promiscuous Plasmid Encoding Resistance to Fluoroquinolones and Third-Generation Cephalosporins. mBio. 2018 Feb 20;9(1):e00105-18. doi: 10.1128/mBio.00105-18. PMID: 29463654; PMCID: PMC5821095.

11. Typhoid fever - Islamic Republic of Pakistan [Internet]. World Health Organization. 2020 [cited 18 April 2020]. Available from: https://www.who.int/csr/don/27-december-2018-typhoid-pakistan/en/

12. Rasheed M, Hasan S, Babar Z, Ahmed S. Extensively drug-resistant typhoid fever in Pakistan. Lancet Infect Dis. 2019;19(3):242-243.

13. Bhatia J, Mathur A, Arora M. Reemergence of Chloramphenicol Sensitivity in Enteric Fever. Med J Armed Forces India. 2007;63(3):212-214.

14. Qamar F, Yousafzai M, Sultana S, Baig A, Shakoor S, Hirani $\mathrm{F}$ et al. A Retrospective Study of Laboratory-Based Enteric Fever Surveillance, Pakistan, 2012-2014. J Infect Dis. 2018;218(suppl_4):S201-S205. 
15. Naheed A, Ram P, Brooks W, Hossain M, Parsons M, Talukder $\mathrm{K}$ et al. Burden of typhoid and paratyphoid fever in a densely populated urban community, Dhaka, Bangladesh. Int J Infect Dis. 2010;14:e93-e99.

16. Laghari G, Hussain Z, Hussain S, Kumar H, Uddin S, Haq A. Antimicrobial Susceptibility Patterns of Salmonella Species in Southern Pakistan. Cureus. 2019;(11)4.

17. Parry C, Ribeiro I, Walia K, Rupali P, Baker S, Basnyat B. Multidrug resistant enteric fever in South Asia: unmet medical needs and opportunities. BMJ. 2019;364:k5322.

18. Britto C, Wong V, Dougan G, Pollard A. A systematic review of antimicrobial resistance in Salmonella enterica serovar Typhi, the etiological agent of typhoid. PLoS Negl Trop Dis. 2018;12(10):e0006779.

19. Golomb B, Koslik H, Redd A. Fluoroquinolone-induced serious, persistent, multisymptom adverse effects. BMJ Case Reports. 2015;bcr2015209821.

20. Chowdhury M, Shumy F, Anam A, Chowdhury M. Current status of typhoid fever : a review. Bangladesh Med J. 2014;43(2):106-111.

21. Bhatia J, Mathur A, Arora M. Reemergence of Chloramphenicol Sensitivity in Enteric Fever. Med J Armed Forces India. 2007;63(3):212-214.

22. Kumar Y, Sharma A, Mani K. Re-emergence of susceptibility to conventionally used drugs among strains of Salmonella Typhi in central west India. J Infect Dev Ctries. 2011;5(03):227-230.
CONFLICT OF INTEREST: Authors declare no conflict of interest

GRANT SUPPORT AND FINANCIAL DISCLOSURE: NIL

\section{AUTHOR'S CONTRIBUTION}

Following authors have made substantial contributions to the manuscript as under

Khan AA: $\quad$ Concept, study design, discussion, man uscript writing, facilitation of the reagent and materials, critical review

Uppal R: $\quad$ Facilitation of the reagent and materials, critical review, interpretation.

Rehan GE: Analysis, interpretation, manuscript writing, study conduction.

Khurshid F: Critical review, study conduction.

Ahmad K: Planning, study conduction, critical review

Zaib H: Study conduction, discussion, manuscript writing

Authors agree to be accountable for all aspects of the work in ensuring that questions related to the accuracy or integrity of any part of the work are appropriately investigated and resolved. 